\title{
Evidence that primer pheromones do not cause social suppression of reproduction in male and female naked mole-rats (Heterocephalus glaber)
}

\author{
C. G. Faulkes ${ }^{1}$ and D. H. Abbott ${ }^{2}$ \\ ${ }^{1}$ Institute of Zoology, Zoological Society of London, Regent's Park, London NW1 4RY, UK; and \\ ${ }^{2}$ Wisconsin Regional Primate Research Centre, 1223 Capitol Court, Madison, WI 53713-1299, USA
}

\begin{abstract}
The role of chemical cues in mediating the reproductive suppression of nonbreeding males and females in colonies of naked mole-rats (Heterocephalus glaber) was examined by comparing reproductive activation in isolated nonbreeders with that of isolated nonbreeders maintained in olfactory and gustatory contact with their parent colony. Animals were either removed from their colony and paired directly with a member of the opposite sex from the same colony, or were removed, housed singly for 40 days, and then paired with a member of the opposite sex from the same parent colony that had also been housed singly for 40 days. Approximately half of these separated animals received a daily transfer of soiled bedding and litter from their parent colony. In females, there was no significant difference between the control and bedding transfer group, in the time taken from separation to the first sustained increase of urinary progesterone above $2 \mathrm{ng} \mathrm{mg}^{-1} \mathrm{Cr}$ indicative of the luteal phase of an ovarian cycle $(7.8 \pm 2.6$ versus $9.4 \pm 2.0$ days, respectively). In both control and bedding transfer groups, singly-housed females commenced ovarian cyclicity, revealing that the presence of a male was not required for reproductive activation and ovulation. In males, concentrations of both urinary testosterone and plasma LH increased after separation from their parent colonies in both control and bedding transfer groups, to reach values equivalent to those of breeding males. The mean time between separation and a rise in urinary testosterone to amounts observed in breeding males $\left(24 \mathrm{ng} \mathrm{mg}^{-1} \mathrm{Cr}\right)$ was not significantly different between control and bedding transfer groups (5.0 \pm 1.4 versus $4.4 \pm 1.7$ days, respectively). There was no significant difference in either plasma LH or urinary testosterone concentrations between the control and bedding transfer groups before or after separation from their parent colonies. These results suggest that primer pheromones from urine, or other secretions contained in soiled bedding and litter, do not play a major role in the suppression of reproduction in nonbreeding male or female naked mole-rats.
\end{abstract}

\section{Introduction}

Primer pheromones, chemical signals released by conspecifics that elicit a physiological response in the recipient animal, are important in modulating reproductive function in many species (for review see Vandenbergh, 1988). Among rodents, the involvement of urinary primer pheromones in suppressing reproduction in both males and females has been well studied. In high density populations of wild mice, Mus musculus (Massey and Vandenbergh, 1980), and bank voles, Clethrionomys glareolus (Kruczek and Marchlewska-Koj, 1986), a urinary pheromone is produced by adult females which delays puberty in juvenile females, thereby slowing down further population growth. In some microtine rodents living in extended family units, suppressing pheromones released by adults may function to reduce reproductive and resource competition by encouraging dispersal of offspring or preventing inbreeding (Batzli et al., 1977; Wolff, 1992).

Received 17 December 1992.
The naked mole-rat, Heterocephalus glaber, exhibits one of the most extreme forms of reproductive suppression of any mammal. These hystricomorph rodents inhabit the arid regions of East Africa, living entirely underground in colonies that often contain $40-90$, and sometimes up to 300 individuals. Their burrow systems contain an extensive network of foraging tunnels up to $2-3 \mathrm{~km}$ in length. These burrows also contain communal nest and toilet chambers, which act as focal points for behavioural and possibly chemosensory communication (Jarvis, 1981, 1985; Brett, 1986, 1991). A characteristic and unique feature of naked mole-rat colonies (which makes them different from other rodent populations) is an extreme behavioural and reproductive division of labour, similar to that of the eusocial insects. Reproduction is restricted to a single breeding female, the 'queen' and one to three males in both captive (Jarvis, 1981; Lacey and Sherman, 1991; Faulkes et al, 1991a) and wild colonies (Jarvis, 1985; Brett, 1991). The remaining colony members do not breed and are reproductively quiescent but not sterile. In nonbreeding females, ovulation is blocked (Faulkes et al., 1990a), apparently as a result of inadequate plasma $\mathrm{LH}$ 
concentrations arising from impaired hypothalamic GnRH secretion (Faulkes et al., 1990b).

Among male naked mole-rats, there are also clear behavioural and physiological differences between breeders and nonbreeders. Only breeding males are solicited by, and mate with, the queen (Jarvis, 1991; Lacey and Sherman, 1991). In addition, breeding males have higher concentrations of plasma $\mathrm{LH}_{\text {, }}$ greater $\mathrm{LH}$ responses to administration of exogenous $\mathrm{GnRH}$, and higher concentrations of urinary testosterone compared with nonbreeders (Faulkes et al., 1991b). In nonbreeding males, although circulating concentrations of reproductive hormones are sufficient to support spermatogenesis (Jarvis, 1991; Faulkes et al., 1991b), most nonbreeding males produce fewer spermatozoa and those produced are nonmotile, compared with breeders (Faulkes et al., in press).

The breeding queen mole-rat attains her position through behavioural domination of all animals in the colony, although the mechanisms by which she suppresses her socially subordinate nonbreeding males and females are unclear. As nonbreeders of both sexes rapidly became reproductively active when removed from their colonies and housed singly, or paired with an animal of the opposite sex, contact with the parent colony is the primary cause of reproductive suppression (Faulkes et al., 1990a; Faulkes and Abbott, 1991). Secretion of primer pheromones by the breeding queen into the communal toilet chamber may be an inhibitory cue operating within naked mole-rat colonies to suppress the reproduction of nonbreeders (Jarvis, 1981; Jarvis and Bennett, 1991).

The aim of this study, therefore, was to investigate the role of primer pheromones present in the communal toilet and nest chamber bedding of parent colonies, in the suppression of reproduction of nonbreeding male and female naked mole-rats separated from their colonies.

\section{Materials and Methods}

\section{Experimental procedures}

Animal housing. Captive colonies of naked mole-rats were maintained at the Institute of Zoology, London, using artificial perspex burrow systems comprising nest, food and toilet chambers interconnected by a series of sealed tunnels, $5 \mathrm{~cm}$ in diameter as described by Faulkes et al. (1990a, b). Separated animals were housed in smaller versions of these 'burrow systems'. All artificial burrow systems were self-contained and enclosed, with gaseous exchange between the colonies and the environment limited to a few holes in the lids of the chambers. Animals were numbered and identified by a system of toe clipping and tattoos. Altogether 11 males and 11 females from four captive colonies were used in this study.

Bedding transfer protocol. Animals were removed from their parent colonies and either (i) paired directly with an individual of the opposite sex from the same colony (control, $n=3$; test, $n=1$ ), or (ii) housed singly for 6 weeks, and then paired with an animal of the opposite sex from the same colony (control $n=3$; test, $n=4$ ). On separation, animals received either a test or control treatment, for periods ranging from 130 to 200 days. From both test groups combined (total test $n=5$ ), separated animals received daily transfer of soiled litter and bedding from the toilet, nest and food chambers from the respective parent colony. A constant volume (approximately $500 \mathrm{ml}$ ) of material was transferred from each chamber in all experiments. Bedding transfer started on the day of separation (designated day 0 ) and continued during the singly housed period and when paired. In the combined control groups $(n=6)$, separated animals were subjected to once daily disturbance of the bedding and litter in their nest, food and toilet chambers, equivalent to that of the bedding transfer group, from day 0 until the end of the experiment.

Monitoring of reproductive activation. Urine samples were collected from test and control animals for periods of up to approximately 60 days before, and up to a maximum of 200 days after, separation from the parent colony. In addition, plasma samples were collected at intervals of approximately 3 weeks before $(n=3)$ and after separation $(n=4-6)$. Routine behavioural observations were not carried out, but casual observations of mating were noted. Urine samples from females were assayed for progesterone as an indicator of ovarian cyclicity, as described by Faulkes et al., 1990a: day 0 of the cycle was designated as the day before a sustained rise in urinary progesterone above $2.0 \mathrm{ng} \mathrm{mg}^{-\mathrm{I}} \mathrm{Cr}$ and was assumed to correspond approximately to the day of ovulation. Day 1 was designated as the start of the luteal phase. Reproductive status in males was monitored by radioimmunoassay of urine samples for testosterone and measurement of bioactive LH. The time taken for males to become reproductively active was measured by defining the latency from separation to a rise in urinary testosterone comparable to those in breeding males as the time in days from separation (day 0 ) to the day on which urinary testosterone concentrations rose from sustained low values to $24 \mathrm{ng} \mathrm{mg}^{-1}$ $\mathrm{Cr}$ (the mean value for breeding males). The latency values were obtained by extrapolating from this value on individual testosterone profiles plotted against time (Faulkes et al., 1991b).

\section{Sampling and radioimmunoassays}

Urine sampling. Urine was chosen for routine steroid hormone determination in males and females in captive colonies in preference to blood to enable regular, repeated sampling from these small animals over several months, and to minimize disturbance to the colonies (Faulkes et al., 1990a, 1991b). Urine sampling involved the removal of all shavings from the toilet chamber in each colony, and wiping the chamber clean with tissue paper. Immediately after each urination, the deposited urine was collected in a glass pipette. After collection of each sample, the toilet chamber was wiped clean with tissue paper. Samples were put on ice immediately, and placed in a freezer within $\mathrm{I} \mathrm{h}$ of collection, and stored at $-20^{\circ} \mathrm{C}$ until hormone determination. Before hormone assay, all urine samples were subjected to a determination of creatinine as described by Bonney et al. (1982). All urinary hormone concentrations were expressed as mass per $\mathrm{mg}$ creatinine $\left(\mathrm{mg}^{-1} \mathrm{Cr}\right.$ ) to correct for dilution of urine.

Blood sampling. Animals were hand-held and the tip of the tail was cut with a sterile scalpel blade and blood (approximately 
$200 \mu \mathrm{l})$ was collected by capillary action using heparinized micro-haematocrit tubes. Blood samples were collected within 2-4 min of animal capture from our captive colonies, and following treatment of the sampling site with an antibiotic powder (Aureomycin Cyanamid, UK), the animal was returned to the colony. Samples were stored on ice for a maximum of $2 \mathrm{~h}$ before they were centrifuged for $5 \mathrm{~min}$ at $500 \mathrm{~g}$, and plasma was stored at $-20^{\circ} \mathrm{C}$ before $\mathrm{LH}$ determination.

Radioimmunoassay of testosterone. Testosterone concentrations were determined in diethyl ether-extracted urine (50$100 \mu \mathrm{l}$ ) by radioimmunoassay following celite chromatography, using sheep anti-testosterone antibody no. 505 (MRC Reproductive Physiology Unit, Edinburgh) as previously described and validated for the naked mole-rat (Faulkes et al., 1991b). The limit of sensitivity of the assay (determined as values with greater than $90 \%$ binding) was $2.0 \mathrm{pg}$ per tube, equivalent to $<1.0 \mathrm{ng} \mathrm{mg}^{-1} \mathrm{Cr}$. Interassay coefficients of variation for repeated determinations of a quality control sample $\left(2.22 \mathrm{ng} \mathrm{mg}^{-1} \mathrm{Cr}\right.$ ), were $15.1 \%(n=4)$ for samples subjected to column chromatography and $16.9 \%(n=4)$ for extracted samples. The intra-assay coefficient of variation was $7.5 \%$ $(n=7)$.

Progesterone radioimmunoassay. Progesterone in female urine was measured in petroleum-ether extracted samples $(50-100 \mu$ l) by radioimmunoassay without chromatography as previously described and validated for the naked mole-rat (Faulkes, 1990; Faulkes et al., 1990a, 1991a). The sensitivity limit of the assay (determined as $90 \%$ binding) was $10 \mathrm{pg}$ per tube. Intra- and interassay precision, expressed as the coefficient of variation for repeated determinations of a quality control, was $6.0 \%(n=11)$ and $7.1 \%(n=19)$, respectively.

Luteinizing hormone bioassay. LH was measured using an in vitro bioassay based on the production of testosterone by dispersed mouse Leydig cells (Van Damme et al., 1974). The method and validation for the naked mole-rat were described by Faulkes (1990) and Faulkes et al. (1990a, b). Plasma samples were assayed in duplicate at two dilutions of 1:10 and 1:20, or $1: 20$ and 1:40, as a routine check for parallelism, and compared with a rat $\mathrm{LH}$ standard over the range $2-0.0625 \mathrm{miu} \mathrm{ml}^{-1}$. The testosterone produced was measured by radioimmunoassay. The sensitivity of the assay (determined by $90 \%$ binding) was $0.1 \mathrm{miu}$ per tube. Intra- and interassay precision for the whole assay, expressed as the mean coefficients of variation for repeated determinations of an LH quality control ( $1.53 \mathrm{miu}$ $\left.\mathrm{ml}^{-1}\right)$, were $10 \%(n=15)$ and $16 \%(n=9)$, respectively.

\section{Statistical analysis}

The times to the first increase of urinary progesterone concentrations were analysed using Student's $t$ test. Plasma LH and urinary testosterone concentrations were analysed using analysis of variance for repeated measures. Comparison of individual means was made post hoc using Duncan's multiple range test (Helwig and Council, 1979).
Table 1. Time after separation of nonbreeding female naked mole-rats from their parent colony to the first sustained increase in urinary progesterone above $2.0 \mathrm{ng} \mathrm{mg}^{-1} \mathrm{Cr}$, indicative of the start of a luteal phase of the ovarian cycle

\begin{tabular}{lcc}
\hline & \multicolumn{2}{c}{$\begin{array}{c}\text { Days to start of } \\
\text { first luteal phase }\end{array}$} \\
\cline { 2 - 3 } Treatment & Control & $\begin{array}{c}\text { Bedding } \\
\text { transfer }\end{array}$ \\
\hline Females removed and paired with & 5 & \\
$\quad$ a male & 18 & 9 \\
Females removed and housed & 7 & 15 \\
singly before pairing & 4 & 8 \\
& 5 & 3 \\
& & 12 \\
Mean \pm SEM & $7.8 \pm 2.6$ & $9.4 \pm 2.0$ \\
& $n=5$ & $n=5$ \\
\hline
\end{tabular}

\section{Results}

Initially, separated animals in both test and control conditions were set up as male-female pairs. However, as the test animals became reproductively active as quickly as the controls, subsequently all separated animals were housed singly at first to eliminate the possibility of the stimulatory effect of a member of the opposite sex overriding pheromonal suppression. Because all of the test animals became reproductively active irrespective of whether they were paired or singly housed, results for both these treatments were combined to increase the sample size.

\section{Females}

Urinary progesterone concentrations could not be detected in all test and control females before day $0\left(<0.5 \mathrm{ng} \mathrm{mg}^{-1} \mathrm{Cr}\right)$, indicating that these females were acyclic nonbreeders before separation to form their colonies. After separation from their parent colonies, bedding transfer and control females were either paired with a male ( $n=1$ and $n=3$, respectively) or were housed singly for six weeks before pairing with a male ( $n=3$ and $n=4$, respectively). All bedding transfer and control groups in either paradigm rapidly commenced ovarian cyclicity. There was no significant difference in the onset of ovarian cyclicity, expressed as the time between separation and the start of the first luteal phase, between the control and bedding transfer groups $(t=0.49 ; P>0.10$; Table 1$)$.

Insufficient urine samples were collected from one control group female to calculate the day of onset of cyclicity, although this individual was seen mating on day 26 after separation, and gave birth to a litter of six pups on day 106. Representative samples of individual urinary progesterone profiles for two females are shown (Fig. I).

Of the six control females, three became pregnant during the course of the experiment, 106, 108 and 110 days respectively, after pairing with a male. Among the six bedding transfer 


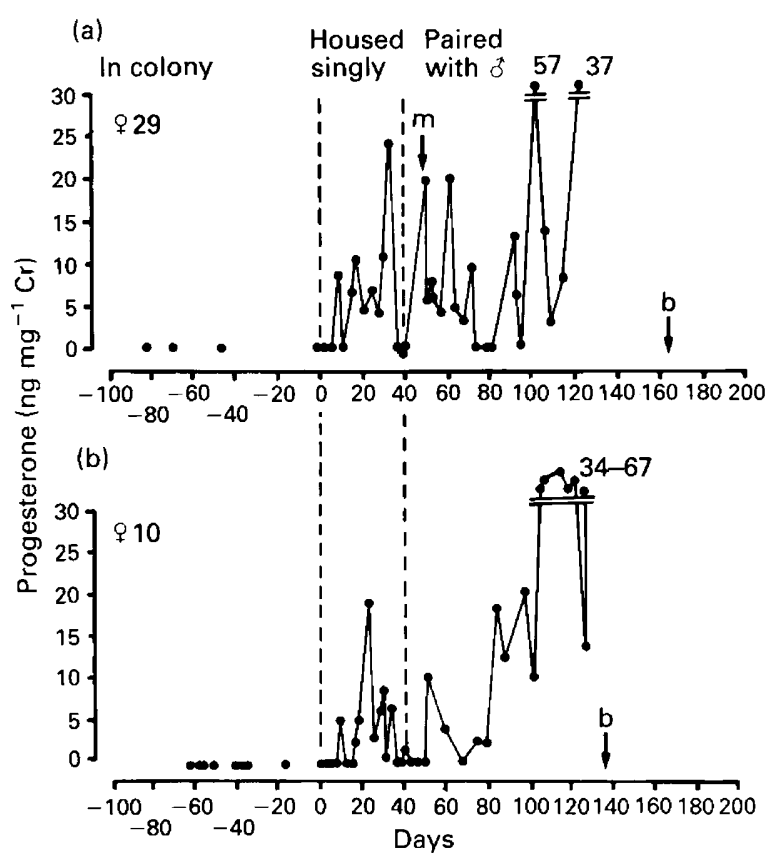

Fig. 1. Urinary progesterone profiles for two female naked mole-rats, while nonbreeding females in their parent colony, when removed and housed singly, and when paired with a nonbreeding male from the same parent colony. The time to the first sustained increase in urinary progesterone above $2 \mathrm{ng} \mathrm{ml}^{-1} \mathrm{Cr}$, indicative of the start of a luteal phase, was 7 days for control female 29 (a) and 15 days for bedding transfer female $10(\mathrm{~b})$. Mating $(\mathrm{m})$ of control female 29 was observed 6 days after the introduction of a male. Both females gave birth (b) 110 and 91 days following the introduction of a male (control female 29 and bedding transfer female 10 , respectively).

females, one died of a gastro-intestinal infection after commencing ovarian cyclicity, and one became pregnant 91 days after pairing with a male. The gestational period of a naked mole-rat is 66-76 days (Jarvis, 1991), and the mean \pm SEM duration of the ovarian cycle was $34.4 \pm 1.6$ days, suggesting that all these births correspond to conception occurring at the second period of oestrus after pairing (e.g. Fig. 1b).

\section{Males}

A total of 302 urine samples (10-45 from each male) and 69 plasma samples (5-9 from each male) were collected. Mean urinary testosterone increased significantly from nonbreeder values (control: $6.5 \pm 2.6 \mathrm{ng} \mathrm{mg}{ }^{-1} \mathrm{Cr}$; bedding transfer: $10.4 \pm 2.3 \mathrm{ng} \mathrm{mg}^{-1} \mathrm{Cr}$ ), after males were separated from their parent colonies in both bedding transfer and control situations $(P<0.01 ; F(2,10)=11.27)$. Urinary testosterone was highest when males were housed singly (control: $49.5 \pm 18.2 \mathrm{ng} \mathrm{mg}^{-1}$ $\mathrm{Cr}$; bedding transfer: $86.8 \pm 25.1 \mathrm{ng} \mathrm{mg}^{-1} \mathrm{Cr}$ ), whereas concentrations decreased to intermediate values when the males were paired with a female (control: $44.5 \pm 25.4 \mathrm{ng} \mathrm{mg}^{-1} \mathrm{Cr}$; bedding transfer: $31.2 \pm 3.5 \mathrm{ng} \mathrm{mg}^{-1} \mathrm{Cr}$ ), a phenomenon that has been discussed previously (Faulkes and Abbott, 1991). There was no significant difference in urinary testosterone between control and bedding transfer groups, either when the males were together in their parent colonies, when housed singly, or when paired with a female $(P=0.23 ; F(1,5)=1.82 ;$ Fig. 2 a).
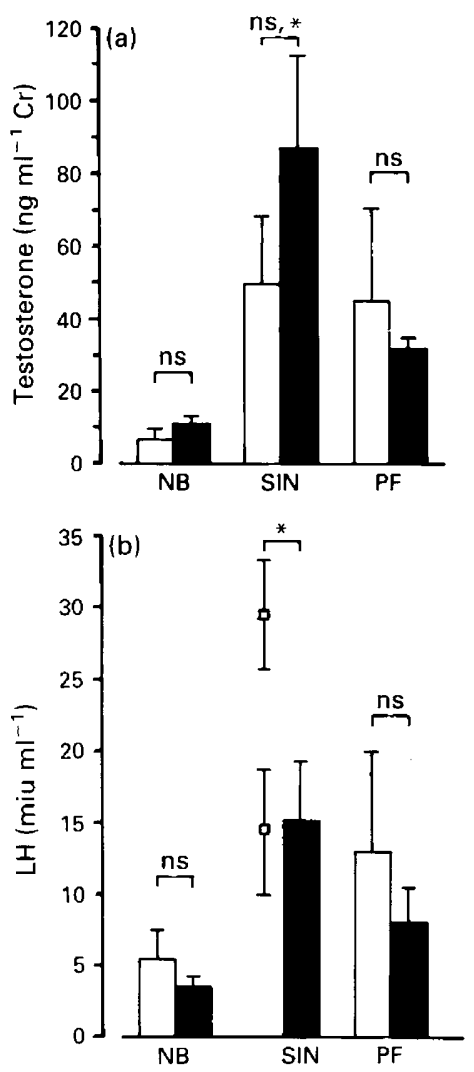

Fig. 2. Concentrations (mean \pm SEM) of (a) urinary testosterone, and (b) plasma $\mathrm{LH}$, in male naked mole-rats when nonbreeders in their parent colonies (NB), when removed from their colonies and housed singly for six weeks (SIN), and when paired with a female (PF). ( $\square$ ) Control; ( $\square$ ) bedding transfer; ${ }^{*} P<0.05$ versus NB and PF; ns: not significant, control versus bedding transfer (Duncan's multiple range test following ANOVA for repeated measures).

Significant increases in concentrations of $\mathrm{LH}$ in plasma occurred when males were removed from their colonies, and followed the same general pattern as that for urinary testosterone concentrations (Fig. 2b). LH concentrations rose from $5.6 \pm 2.2 \mathrm{miu} \mathrm{ml}^{-1}$ (control) and $3.5 \pm 0.7 \mathrm{miu} \mathrm{ml}^{-1}$ (bedding transfer), to significantly higher concentrations when males were housed singly $(P<0.01 ; F(2,8)=11.32$; bedding transfer: $15.2 \pm 4.2$; control: plasma samples were only obtained from two males, therefore their individual means \pm SEM of $14.4 \pm$ 4.7 and $29.6 \pm 3.7$ are displayed in Fig. $2 b$, rather than means for three or more males). There was no significant difference in plasma LH between control and bedding transfer groups, either when the males were together in their parent colonies, or when paired with a female $(P=0.88 ; F(1,4)=0.02$; Fig. 2 b). When housed singly, the mean concentrations of $\mathrm{LH}$ in plasma obtained from two of the control males were comparable to those of the bedding transfer males.

From examination of individual urinary testosterone profiles, the mean time between separation and a rise in urinary testosterone to $24 \mathrm{ng} \mathrm{mg}^{-1} \mathrm{Cr}$, the mean for breeding males was calculated as $5.0 \pm 1.4$ and $4.4 \pm 1.7$ days, for control and bedding transfer males, respectively $(t=0.27 ; P>0.10$; Table 2$)$. One control group male was not included in the calculation as 
Table 2. Time after separation of nonbreeding male naked molerats from their parent colony to the first sustained increase of urinary testosterone to $24 \mathrm{ng} \mathrm{mg}^{-1} \mathrm{Cr}$, the mean value of breeding males

\begin{tabular}{lcc}
\hline & $\begin{array}{c}\text { Days to increase of } \\
\text { urinary testosterone }\end{array}$ & $\begin{array}{c}\text { Bedding } \\
\text { transfer }\end{array}$ \\
\cline { 2 - 3 } Treatment & Control & \\
\hline Males removed and paired with & 9 & 9 \\
$\quad$ a female & 1 & 1 \\
Males removed and housed singly & 7 & 3 \\
before pairing & 5 & 1 \\
& 3 & 8 \\
Mean \pm SEM & & $4.4 \pm 1.7$ \\
& $5.0 \pm 1.4$ & $n=5$ \\
\hline
\end{tabular}

insufficient urine samples were collected for this individual during the early period of separation.

\section{Discussion}

The suppression of reproduction in nonbreeding male and female naked mole-rats occurs as a direct result of inhibitory factors operating within their social environment, because nonbreeding animals removed from their parent colonies and either housed singly or placed together as male-female pairs rapidly became reproductively active. Reproductive activation in separated male and female naked mole-rats was neither robustly delayed, nor prevented by the daily transfer of soiled litter and bedding from the nest, food and toilet chambers from their parent colonies, material which should potentially contain both volatile and nonvolatile chemosignals. Thus, a primer pheromone alone does not appear to be a major factor in suppressing reproduction in nonbreeding naked mole-rats, and a behavioural mechanism is implicated. Similar experimental designs to that used in this study, involving transfer of soiled bedding or litter, have successfully demonstrated suppressing pheromones in several other rodent species. These include delay of sexual maturation in female pine voles, Microtus pinetorum (Lepri and Vandenbergh, 1986), and white-footed mice, Peromyscus leucopus (Rogers and Beauchamp, 1976), and puberty delay in female mice (Drickamer, 1974), an effect subsequently shown to be mediated by volatile urinary pheromones (Jemiolo et al., 1986).

In social mammals that exhibit co-operative behaviour and reproductive suppression, behavioural interactions between dominant breeders and subordinate nonbreeding group members appear to be an important factor in imposing suppression. Such behavioural interactions may simply take the form of an interference by dominants with normal patterns of courtship and mating in subordinates, for example male dwarf mongoose, Helogale parvula (Creel et al., 1992), whereas in other cases, behavioural cues in the form of agonistic interactions may be translated into a physiological block to reproduction, for example female dwarf mongoose (Creel et al., 1992). In some marmoset and tamarin monkeys, both behavioural and olfactory signals are implicated in reproductive suppression. A delay in the onset of ovulation in subordinate females removed from their social groups but maintained in scent contact, has been demonstrated in both the cotton top tamarin, Saguinus oedipus (Savage et al., 1988), and the common marmoset monkey Callithrix jacchus (Barrett et al., 1990). Subordinate female common marmosets kept in behavioural contact with their social groups but rendered anosmic do not become reproductively active, suggesting that behavioural and visual cues may override the suppressing influence of odour (Barrett et al., 1993).

In the naked mole-rat, the hypothesis that behavioural cues from the breeding female are the main factor in mediating reproductive suppression is consistent with observations of queen succession. If a queen dies or is removed from her colony, then suppression is removed and several nonbreeding females will become reproductively active. Fighting normally (but not always) occurs among these females until the most dominant animal takes over, often after killing rivals (Jarvis, 1991; Lacey and Sherman, 1991). Furthermore, breeding queens may also occasionally be killed and replaced by a nonbreeding female that becomes both reproductively active and behaviourally dominant (Faulkes, 1990). Such evidence argues against a pheromonal cue from the breeding female, as it is behavioural agonistic competition that results in the deposing of a queen or in the final determination of queen succession. An inhibitory primer pheromone would preclude the importance of behavioural interactions in determining breeding status.

While behavioural contact with the breeding queen appears to be the most important factor in mediating reproductive suppression, individual specific odours may act as both recognition cues (naked mole-rats are blind), and reinforcing cues for behaviour. Thus, the odour of the queen may emphasize her presence within the colony, but in her absence, these odour cues alone are not sufficient to maintain suppression of reproduction. Characteristic autogrooming is observed when animals use the communal toilet chamber (Lacey et al., 1991), and it has been suggested that the function of this behaviour is to transfer suppressing pheromones from the breeding queen to the nonbreeders. However, the autogrooming behaviour may also, or alternatively, function to maintain both individual-specific and colony-specific odour on each animal, for recognition purposes. Behavioural studies involving two-choice $Y$-maze tests have shown that naked mole-rats can discriminate between their own and foreign colony toilet chamber odours (Faulkes, 1990).

The nature of the behavioural cues that mediate reproductive suppression in naked mole-rats remain unknown, but presumably involve agonistic interactions between the queen and the nonbreeders of both sexes, possibly resulting in a stress-induced inhibition of fertility. It is likely that after a female has attained the position of the dominant breeding queen in a colony, she may communicate her status and maintain suppression in her subordinates by agonistic behavioural interactions. Perhaps the most overtly aggressive behaviour commonly observed in naked mole-rat colonies is shoving, whereby one animal pushes another during a 'head to head' meeting (Lacey et al., 1991). Shoving is almost exclusively initiated by the breeding queen, although it is occasionally observed in breeding males, and 
tends to be directed more at larger individuals within the colony, regardless of their sex (Reeve and Sherman, 1991). It is possible that shoving is one behaviour involved in imposing reproductive suppression, and the fact that the queen shoves the breeding male, and both sexes of nonbreeders, is consistent with our results suggesting that the queen influences the reproductive physiology of females (Faulkes et al., 1990a), and breeder and nonbreeder males (Faulkes and Abbott, 1991). In conclusion, our study demonstrates that primer pheromonal influences on reproduction, previously described in other rodents, do not appear to be a major factor in the suppression of reproduction in naked mole-rat colonies, and therefore a behavioural mechanism may predominate.

The authors thank M. J. Llovett and the laboratory animal staff at the Institute of Zoology for care and maintenance of the animals, A. P. F. Flint and A. S. I. Loudon for criticism of the manuscript, and T. Dennett and M. J. Walton for preparation of the figures. This work was supported by an MRC/AFRC Programme grant and a grant from the Wellcome Trust (to D. H. Abbott) and an SERC Research Studentship (to C. G. Faulkes).

\section{References}

Barrett J, Abbott DH and George LM (1990) Extension of reproductive suppression by pheromonal cues in subordinate female marmoset monkeys, Callithrix jacchus Journal of Reproduction and Fertility 90 411-418

Barrett J, Abbott DH and George LM (1993) Sensory cues and the suppression of reproduction in subordinate female marmoset monkeys, Callithrix jacchus Joumal of Reproduction and Fertility 97 301-310

Batzli GO, Getz LL and Hurley SS (1977) Suppression of growth and reproduction of microtine rodents by social factors Journal of Mammology 58 583-591

Bonney RC, Wood DJ and Kleiman DG (1982) Endocrine correlates of behavioural oestrus in the female giant panda (Ailuropoda melanoleuca) and associated hormonal changes in the male journal of Reproduction and Fertility 64 209-215

Brett RA (1986) Ecology and Behaviour of the Naked Mole-rat Heterocephalus glaber (Ruppell) Rodentia: Bathyergidae. PhD thesis, University of London

Brett RA (1991) The ecology of naked mole-rat colonies: burrowing, food, and limiting factors. In The Biology of the Naked Mole-Rat pp 137-184 Eds PW Sherman, JUM Jarvis and RD Alexander. Princeton University Press, New York

Creel S, Creel N, Wildt DE and Monfort SL (1992) Behavioural and endocrine mechanisms of reproductive suppression in Serengeti dwarf mongooses Animal Behaviour 43 231-245

Drickamer LC (1974) Sexual maturation of female house mice: social inhibition Developmental Psychobiology 7 257-265

Faulkes CG (1990) Social Suppression of Reproduction in the Naked Mole-rat, Heterocephalus glaber. PhD thesis, University of London

Faulkes CG and Abbott DH (1991) Social control of reproduction in breeding and non-breeding male naked mole-rats (Heterocephalus glaber) Journal of Reproduction and Fertility 93 427-435

Faulkes CG, Abbott DH and Jarvis JUM (1990a) Social suppression of ovarian cyclicity in captive and wild colonies of naked mole-rats, Heterocephalus glaber Joumal of Reproduction and Fertility 88 559-568

Faulkes CG, Abbott DH, Jarvis JUM and Sherriff F (1990b) LH responses of female naked mole-rats, Heterocephalus glaber, to single and multiple doses of exogenous GnRH Joumal of Reproduction and Fertility 89 317-323
Faulkes CG, Abbott DH, Liddell CE, George LM and Jarvis JUM (1991a) Hormonal and behavioural aspects of reproductive suppression in female naked mole-rats Heterocephalus glaber. In The Biology of The Naked Mole-Rat pp 426-445 Eds PW Sherman, JUM Jarvis and RD Alexander. Princeton University Press, New York

Faulkes CG, Abbott DH and Jarvis JUM (1991b) Social suppression of reproduction in male naked mole-rats, Heterocephalus glaber journal of Reproduction and Fertility 91 593-604

Faulkes CG, Trowell SN and Taggart DA Investigation of sperm numbers and motility in reproductivity active and socially suppressed naked mole-rats, Heterocephalus glaber. In Proceedings of the Second International Conference on Advances in Reproductive Research in Man and Animals Ed. CS Bambra. Institute of Primate Research, National Museums of Kenya (in press)

Helwig JT and Council KA (1979) SAS Users Guide SAS Institute Inc, Cary

Jarvis JUM (1981) Eu-sociality in a mammal - cooperative breeding in naked mole-rat Heterocephalus glaber colonies Science 212 571-573

Jarvis JUM (1985) Ecological studies on Heterocephalus glaber, the naked mole-rat, in Kenya National Geographic Scientific Report 20 429-437

Jarvis JUM (1991) Reproduction. In The Biology of The Naked Mole-Rat pp 384425 Eds PW Sherman, JUM Jarvis and RD Alexander. Princeton University Press, New York

Jarvis JUM and Bennett NC (1991) Ecology and behaviour of the family Bathyergidae. In The Biology of The Naked Mole-Rat pp 66-96 Eds PW Sherman, JUM Jarvis and RD Alexander. Princeton University Press, New York

Jemiolo B, Andreolini F and Novotny M (1986) Chemical and biological investigations of female mouse pheromones. In Chemical Signals In Vertebrates 4: Ecology, Evolution And Comparative Biology pp 79-85 Eds D Duvall, D Muller-Schwarze and RM Silverstein. Plenum Press, New York

Kruczek M and Marchlewska-Koj AM (1986) Puberty delay of bank vole females in a high-density population Biology of Reproduction 35 537-541

Lacey EA and Sherman PW (1991) Social organization of naked mole-rat (Heterocephalus glaber) colonies: evidence for a division of labour. In The Biology of the Naked Mole-Rat pp 275-336 Eds JUM Jarvis, PW Sherman and RD Alexander. Princeton University Press, New York

Lacey EA, Pepper JW, Alexander RD, Braude SH, Sherman PW and Jarvis JUM (1991) An ethogram for the naked mole-rat: non-vocal behaviours. In The Biology of the Naked Mole-Rat pp 209-242 Eds PW Sherman, JUM Jarvis and RD Alexander. Princeton University Press, New York

Lepri JJ and Vandenbergh JG (1986) Puberty in pine voles, Microtus pinetorum, and the influence of chemosignals on female reproduction Biology of Reproduction 34 370-377

Massey A and Vandenbergh JG (1980) Puberty delay by a urinary cue from female house mice in feral populations Science 209 821-822

Reeve HK and Sherman PW (1991) Intracolonial aggression and nepotism by the breeding female naked mole-rat. In The Biology Of The Naked Mole-Rat pp 337-357 Eds JUM Jarvis, PW Sherman and RD Alexander. Princeton University Press, New York

Rogers JG, Jr and Beauchamp GK (1976) Influence of stimuli from populations of Peromyscus leucopus on maturation of young Journal of Mammalogy 57 $320-330$

Savage A, Ziegler TE and Snowdon CT (1988) Sociosexual development, par bond formation and mechanisms of fertility suppression in female cotton-top tamarins (Saguinus oedipus oedipus) American Journal of Primatology 14 $345-359$

Van Damme M-P, Robertson DM and Diczfalusy E (1974) An improved in vitro bioassay method for measuring luteinizing hormone (LH) activity using mouse Leydig cell preparations Acta Endocrinologica Copenhagen 77 655-671

Vandenbergh JG (1988) Pheromones and mammalian reproduction. In The Physiology of Reproduction pp 1679-1695 Eds E Knobil and JA Neill. Raven Press Ltd, New York

Wolff JO (1992) Parents suppress reproduction and stimulate dispersal in opposite-sex juvenile white-footed mice Nature 359 409-410 